\title{
Quantum phases in an optical lattice
}

\author{
D. van Oosten, ${ }^{1,2}$ P. van der Straten, ${ }^{2}$ and H. T. C. Stoof $^{1}$ \\ ${ }^{1}$ Institute for Theoretical Physics, University of Utrecht, Princetonplein 5, 3584 CC Utrecht, The Netherlands \\ ${ }^{2}$ Debye Institute, University of Utrecht, Princetonplein 5, 3584 CC Utrecht, The Netherlands
}

(Received 6 November 2000; published 12 April 2001)

\begin{abstract}
We present the zero-temperature phase diagram of bosonic atoms in an optical lattice, using two different mean-field approaches. The phase diagram consists of various insulating phases and a superfluid phase. We explore the nature of the insulating phase by calculating both the quasiparticle and quasihole dispersion relation. We also determine the parameters of our single band Bose-Hubbard model in terms of the microscopic parameters of the atoms in the optical lattice.
\end{abstract}

DOI: 10.1103/PhysRevA.63.053601

PACS number(s): 03.75.Fi, 67.40.-w, 32.80.Pj, 39.25.+k

\section{INTRODUCTION}

Using the interference pattern of intersecting laser beams one can create a periodic potential for atoms, which is known as an optical lattice [1,2]. Because one can confine atoms at separate lattice sites, one can accurately control the interaction between the atoms. This makes the optical lattice an important tool in spectroscopy, laser cooling [3], and quantum computing [4]. In the following we study some of the many-body aspects of such a lattice and in particular BoseEinstein condensation of atoms in a optical lattice. In contrast with the existing Bose-Einstein condensation experiments in an harmonic trap, the quantum depletion of the condensate in the case of Bose-Einstein condensation in a lattice can be very large. We can therefore expect interesting features.

If we assume that the atoms are cooled to within the lowest Bloch band of the periodic potential, Jaksch et al. [5] have shown that we can describe the behavior of the atoms in an optical lattice with the Bose-Hubbard Hamiltonian

$$
H=-t \sum_{\langle i, j\rangle} c_{i}^{\dagger} c_{j}+\frac{1}{2} U \sum_{i} c_{i}^{\dagger} c_{i}^{\dagger} c_{i} c_{i}-\mu \sum_{i} c_{i}^{\dagger} c_{i},
$$

where the sum in the first term on the right-hand side is restricted to nearest neighbors and $c_{i}^{\dagger}$ and $c_{i}$ are the creation and annihilation operators of an atom at site $i$, respectively. The parameter $t$ is the hopping parameter and $U$ is the interaction strength, which we always assume to be positive in the following. The term involving the chemical potential $\mu$ is added because we perform our calculations in the grandcanonical ensemble.

Qualitatively we expect that when there is an integer number of particles at each site $i$ and $t \ll U$, the interaction between the particles will make it energetically unfavorable for a particle to move from one site to another. In this situation the gas is in what is known as the Mott insulator phase [6]. However, if we add in this phase a particle to the system, this particle will only receive a small energetic penalty when it moves, because its interaction energy is the same on each site. For this reason, a gas with a noninteger number of bosons at each site will be in a superfluid phase at zero temperature. This expectation has been shown to be correct using quantum Monte Carlo calculations [7] and several mean-field approaches $[5,8,9]$. In particular, Ref. [5] numerically determines interesting features of cold bosonic atoms in an inhomogeneous optical lattice. In this paper, we give a largely analytical means of understanding the results obtained by these authors.

In order to describe the zero-temperature phase transition from the superfluid to the Mott-insulating phase analytically, we need to make some appropriate mean-field approximation to the Hamiltonian in Eq. (1). A more or less standard approach would be to use the Bogoliubov approximation. In Sec. II we show that this approximation does not predict the expected phase transition and we explain the absence of the phase transition. In Sec. III we analytically investigate an alternative mean-field theory, proposed by Sheshadri et al. [9], and compare the analytical results with exact numerical results. In Sec. IV we discuss the properties of the Mott insulating phases by calculating the quasiparticle and quasihole dispersions and finally in Sec. V we relate the parameters $t$ and $U$ to experimental parameters such as laser intensity and wavelength.

\section{BOGOLIUBOV APPROXIMATION}

We first transform the Hamiltonian to momentum space by introducing creation and annihilation operators $a_{\mathbf{k}}^{\dagger}$ and $a_{\mathbf{k}}$ respectively, such that

$$
\begin{gathered}
c_{i}=\frac{1}{\sqrt{N_{s}}} \sum_{\mathbf{k}} a_{\mathbf{k}} e^{-i \mathbf{k} \cdot \mathbf{r}_{i}}, \\
c_{i}^{\dagger}=\frac{1}{\sqrt{N_{s}}} \sum_{\mathbf{k}} a_{\mathbf{k}}^{\dagger} e^{i \mathbf{k} \cdot \mathbf{r}_{i}},
\end{gathered}
$$

where $N_{s}$ is the number of lattice site and $\mathbf{r}_{i}$ is the coordinate of site $i$. The wave vector $\mathbf{k}$ runs only over the first Brillouin zone. For mathematical convenience we take only a finite volume $V$, so that the momenta $\hbar \mathbf{k}$ are discretized, which allows us to write sums instead of integrals in Eq. (2). Later we will take the continuum limit $V \rightarrow \infty$. Using the fact that $\sum_{i} e^{-i\left(\mathbf{k}-\mathbf{k}^{\prime}\right) \cdot \mathbf{r}_{i}}=N_{s} \delta_{\mathbf{k}, \mathbf{k}^{\prime}}$, it is easily shown that the prefactor $1 / \sqrt{N_{s}}$ ensures that the total number of particles obeys $N$ $=\Sigma_{i} c_{i}^{\dagger} c_{i}=\Sigma_{\mathbf{k}} a_{\mathbf{k}}^{\dagger} a_{\mathbf{k}}$. 
If we limit our description to cubic lattices with lattice distance $a$ and substitute Eq. (2) into the Hamiltonian, we find

$$
\begin{aligned}
H= & \sum_{\mathbf{k}}\left(-\bar{\epsilon}_{\mathbf{k}}-\mu\right) a_{\mathbf{k}}^{\dagger} a_{\mathbf{k}} \\
& +\frac{1}{2} \frac{U}{N_{s}} \sum_{\mathbf{k}} \sum_{\mathbf{k}^{\prime}} \sum_{\mathbf{k}^{\prime \prime}} \sum_{\mathbf{k}^{\prime \prime \prime}} a_{\mathbf{k}}^{\dagger} a_{\mathbf{k}^{\prime}}^{\dagger} a_{\mathbf{k}^{\prime \prime}} a_{\mathbf{k}^{\prime \prime \prime}} \delta_{\mathbf{k}+\mathbf{k}^{\prime}, \mathbf{k}^{\prime \prime}+\mathbf{k}^{\prime \prime \prime}},
\end{aligned}
$$

where we defined $\bar{\epsilon}_{\mathbf{k}}=2 t \sum_{j=1}^{d} \cos \left(k_{j} a\right)$, with $d$ the number of dimensions. For a Bose condensed gas the average number of condensate atoms $N_{0}$ is a number much larger then one, which means that $N_{0}=\left\langle a_{\mathbf{0}}^{\dagger} a_{\mathbf{0}}\right\rangle \approx\left\langle a_{\mathbf{0}} a_{\mathbf{0}}^{\dagger}\right\rangle$ and we are allowed to take $N_{0}=\left\langle a_{\mathbf{0}}^{\dagger}\right\rangle\left\langle a_{\mathbf{0}}\right\rangle$.

Since $\left\langle a_{\mathbf{0}}^{\dagger}\right\rangle$ and $\left\langle a_{\mathbf{0}}\right\rangle$ are complex conjugates, we conclude that $\left\langle a_{\mathbf{0}}^{\dagger}\right\rangle=\left\langle a_{\mathbf{0}}\right\rangle=\sqrt{N_{0}}$, where we have chosen these expectation values to be real. The Bogoliubov approach consists of replacing the creation and annihilation operators by their average $\sqrt{N_{0}}$ plus a fluctuation

$$
\begin{aligned}
& a_{\mathbf{0}}^{\dagger} \rightarrow \sqrt{N_{0}}+a_{\mathbf{0}}^{\dagger}, \\
& a_{\mathbf{0}} \rightarrow \sqrt{N_{0}}+a_{\mathbf{0}},
\end{aligned}
$$

and minimizing the energy of the gas with respect to the number of condensate atoms $N_{0}$. At the minimum, the part of the Hamiltonian that is linear in the fluctuations must therefore be zero. Performing the above substitution and selecting the linear terms yields

$$
H^{(1)}=\left(-\bar{\epsilon}_{\mathbf{0}}-\mu+\frac{U}{N_{s}} N_{0}\right) \sqrt{N_{0}}\left(a_{\mathbf{0}}^{\dagger}+a_{\mathbf{0}}\right),
$$

where the superscript denotes the order in the fluctuations. Since $H^{(1)}$ must be zero for all $a_{0}^{\dagger}$ and $a_{0}$ we conclude that in the lowest-order approximation

$$
\mu=U n_{0}-z t,
$$

in terms of the condensate density $n_{0}=N_{0} / N_{s}$ and the number of nearest neighbors $z=2 d$. This expression can be easily understood since the chemical potential is the energy needed to add one particle to the system. Adding one particle results in an energy increase due to the interaction with the $n_{0}$ particles already at each site, and an energy decrease due to the possible hopping to one of $z$ nearest-neighbor sites.

Next we determine the effective hamiltonian $H^{\text {eff }}$, which contains only the parts of zeroth and second order in the fluctuations. The zeroth-order term is found by substituting all creation and annihilation operators by $\sqrt{N_{0}}$. To find the quadratic term, we substitute in the interaction term two creation or annihilation operators at a time by $\sqrt{N_{0}}$ and write down all possible combinations. Performing the summation over one of the remaining momenta yields finally

$$
\begin{aligned}
H^{\mathrm{eff}}= & \left(-z t-\mu+\frac{1}{2} U n_{0}\right) N_{0}+\sum_{\mathbf{k}}\left(-\bar{\epsilon}_{\mathbf{k}}-\mu\right) a_{\mathbf{k}}^{\dagger} a_{\mathbf{k}} \\
& +\frac{1}{2} U n_{0} \sum_{\mathbf{k}}\left(a_{\mathbf{k}} a_{-\mathbf{k}}+4 a_{\mathbf{k}}^{\dagger} a_{\mathbf{k}}+a_{-\mathbf{k}}^{\dagger} a_{\mathbf{k}}^{\dagger}\right) .
\end{aligned}
$$

We can simplify this expression somewhat by using the commutation relation $\left[a_{\mathbf{k}}, a_{\mathbf{k}}^{\dagger}\right]=1$. If we also substitute Eq. (6) and write $\epsilon_{\mathbf{k}}=z t-\bar{\epsilon}_{\mathbf{k}}$, we find

$$
\begin{aligned}
H^{\mathrm{eff}}= & -\frac{1}{2} U n_{0} N_{0}-\frac{1}{2} \sum_{\mathbf{k}}\left(\epsilon_{\mathbf{k}}+U n_{0}\right)+\frac{1}{2} \sum_{\mathbf{k}}\left(a_{\mathbf{k}}^{\dagger}, a_{-\mathbf{k}}\right) \\
& \times\left[\begin{array}{cc}
\epsilon_{\mathbf{k}}+U n_{0} & U n_{0} \\
U n_{0} & \epsilon_{\mathbf{k}}+U n_{0}
\end{array}\right]\left(\begin{array}{c}
a_{\mathbf{k}} \\
a_{-\mathbf{k}}^{\dagger}
\end{array}\right)
\end{aligned}
$$

where the extra zeroth-order terms are generated by the commutation of $a_{\mathbf{k}}^{\dagger}$ and $a_{\mathbf{k}}$.

The effective Hamiltonian is diagonalized by a Bogoliubov transformation. This implies that we define new creation and annihilation operators $b_{\mathbf{k}}^{\dagger}$ and $b_{\mathbf{k}}$ for which the effective Hamiltonian is diagonal, by means of

$$
\left(\begin{array}{c}
b_{\mathbf{k}} \\
b_{-\mathbf{k}}^{\dagger}
\end{array}\right)=\left[\begin{array}{cc}
u_{\mathbf{k}} & v_{\mathbf{k}} \\
v_{\mathbf{k}}^{*} & u_{\mathbf{k}}^{*}
\end{array}\right]\left(\begin{array}{c}
a_{\mathbf{k}} \\
a_{-\mathbf{k}}^{\dagger}
\end{array}\right) \equiv \mathbf{B}\left(\begin{array}{c}
a_{\mathbf{k}} \\
a_{-\mathbf{k}}^{\dagger}
\end{array}\right) .
$$

To ensure that the operators $b_{\mathbf{k}}^{\dagger}$ and $b_{\mathbf{k}}$ still obey the standard commutation relations for bosonic creation and annihilation operators, we have to demand that the coefficients of the matrix B obey

$$
\left|u_{\mathbf{k}}\right|^{2}-\left|v_{\mathbf{k}}\right|^{2}=1 .
$$

If we now substitute Eq. (9) into Eq. (8) and demand that the result reduces to the diagonal Hamiltonian

$$
\begin{aligned}
H^{\mathrm{eff}}= & -\frac{1}{2} U n_{0} N_{0}+\frac{1}{2} \sum_{\mathbf{k}}\left[\hbar \omega_{\mathbf{k}}-\left(\epsilon_{\mathbf{k}}+U n_{0}\right)\right] \\
& +\sum_{\mathbf{k}} \hbar \omega_{\mathbf{k}} b_{\mathbf{k}}^{\dagger} b_{\mathbf{k}},
\end{aligned}
$$

we find that $u_{\mathbf{k}}$ and $v_{\mathbf{k}}$ must be solutions of the following two equations:

$$
\begin{gathered}
{\left[\left(u_{\mathbf{k}}\right)^{2}+\left(v_{\mathbf{k}}\right)^{2}\right] U n_{0}-2 u_{\mathbf{k}} v_{\mathbf{k}}\left(\epsilon_{\mathbf{k}}+U n_{0}\right)=0,} \\
\left(\left|u_{\mathbf{k}}\right|^{2}+\left|v_{\mathbf{k}}\right|^{2}\right)\left(\epsilon_{\mathbf{k}}+U n_{0}\right)-\left(u_{\mathbf{k}}^{*} v_{\mathbf{k}}+u_{\mathbf{k}} v_{\mathbf{k}}^{*}\right) U n_{0}=\hbar \omega_{\mathbf{k}} .
\end{gathered}
$$

Using the normalization in Eq. (10), we can easily find the solution

$$
\begin{gathered}
\hbar \omega_{\mathbf{k}}=\sqrt{\epsilon_{\mathbf{k}}^{2}+2 U n_{0} \epsilon_{\mathbf{k}}}, \\
\left|v_{\mathbf{k}}\right|^{2}=\left|u_{\mathbf{k}}\right|^{2}-1=\frac{1}{2}\left(\frac{\epsilon_{\mathbf{k}}+U n_{0}}{\hbar \omega_{\mathbf{k}}}-1\right) .
\end{gathered}
$$


To also obtain the condensate density $n_{0}$, which until now has been arbitrary, we now need to calculate the total density $n$ as given by our effective Hamiltonian. The total density is thus given by

$$
n=\frac{1}{N_{s}} \sum_{\mathbf{k}}\left\langle a_{\mathbf{k}}^{\dagger} a_{\mathbf{k}}\right\rangle_{H^{\mathrm{eff}},}
$$

where the brackets \langle\rangle$_{H^{\text {eff }}}$ denote the expectation value as calculated with the effective Hamiltonian $H^{\text {eff }}$. For a Bosecondensed gas, this density consists of two parts: the density associated with the macroscopic occupation of the oneparticle ground state, i.e., the condensate, and the density due to the occupation of the higher lying one-particle states. In this case, the condensate density equals the parameter $n_{0}$ and the density of the noncondensate part is determined by an average over the quadratic fluctuations, which will be a function of $n_{0}$. Calculating the average over the quadratic fluctuations by means of Eq. (9) yields first of all

$$
n=n_{0}+\frac{1}{N_{s}} \sum_{\mathbf{k} \neq 0}\left[\left(\left|u_{\mathbf{k}}\right|^{2}+\left|v_{\mathbf{k}}\right|^{2}\right)\left\langle b_{\mathbf{k}}^{\dagger} b_{\mathbf{k}}\right\rangle_{H^{\mathrm{eff}}}+\left|v_{\mathbf{k}}\right|^{2}\right] .
$$

If we then use Eq. (13) and substitute the Bose distribution evaluated at $\hbar \omega_{\mathbf{k}}$ for $\left\langle b_{\mathbf{k}}^{\dagger} b_{\mathbf{k}}\right\rangle_{H^{\text {eff }}}$ we find that

$$
n=n_{0}+\frac{1}{N_{s}} \sum_{\mathbf{k} \neq 0}\left(\frac{\epsilon_{\mathbf{k}}+U n_{0}}{\hbar \omega_{\mathbf{k}}} \frac{1}{e^{\beta \hbar \omega_{\mathbf{k}}-1}}+\frac{\epsilon_{\mathbf{k}}+U n_{0}-\hbar \omega_{\mathbf{k}}}{2 \hbar \omega_{\mathbf{k}}}\right) .
$$

In the zero-temperature limit, $\beta \rightarrow \infty$, the first term in the summand is zero. Taking the continuum limit by using $\Sigma_{\mathbf{k}}$ $\rightarrow V \int_{-\pi / a}^{\pi / a} d \mathbf{k} /(2 \pi)^{d}$, changing from momenta $\mathbf{k}$ to $\mathbf{q}$ $=2 \pi \mathbf{k} / a$, and realizing that $N_{s}=V / a^{d}$, we arrive at the expression

$$
n=n_{0}+\frac{1}{2} \int_{-1 / 2}^{1 / 2} d \mathbf{q}\left(\frac{\epsilon_{\mathbf{q}}+U n_{0}}{\hbar \omega_{\mathbf{q}}}-1\right)
$$

with $\quad \epsilon_{\mathbf{q}}=2 t \sum_{j=1}^{d}\left[1-\cos \left(2 \pi q_{j}\right)\right] \quad$ and $\quad \hbar \omega_{\mathbf{q}}=\left(\epsilon_{\mathbf{q}}^{2}\right.$ $\left.+2 U n_{0} \epsilon_{\mathrm{q}}\right)^{1 / 2}$. We can now obtain the condensate density by solving Eq. (17) for $n_{0}$ for a fixed value of $n$. We expect that at integer $n$, for a fixed value of $U / t$, there will be no superfluid solution and this will mark the phase transition to the insulating phase as predicted by Refs. [5,7-9].

\section{A. Numerical results}

In Fig. 1(a) we plotted the result of this calculation for a two-dimensional lattice. We see from this figure, that there is only a marginal difference between the case that $n=0.5$ and $n=1.0$. In Fig. 1(b) we plotted the result for a threedimensional lattice. In this case the difference between halffilling and integer filling is somewhat larger, but there is clearly no critical value of $U / t$ for which the condensate density goes to zero.

These results lead to the suspicion that the phase transition to the insulating phase is not present in this approxima-

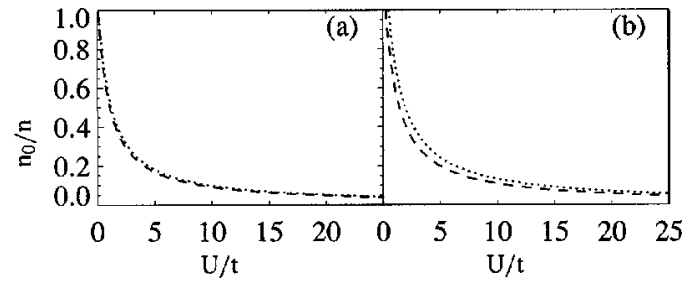

FIG. 1. The condensate fraction $n_{0} / n$ (a) in a two-dimensional optical lattice and (b) a three-dimensional optical lattice, both as a function of the dimensionless parameter $U / t$ for $n=0.5$ (dashed line) and $n=1.0$ (dotted line).

tion. To verify this, we investigate the limit of $U / t \rightarrow \infty$ in some detail.

\section{B. Asymptotic behavior}

When $U / t \rightarrow \infty$ we intuitively expect the system to become an insulator, because it effectively means that the hopping parameter goes to zero. We therefore expect that there are no superfluid solutions as $U / t \rightarrow \infty$. We can see that in this limit the integrand in the right-hand side of Eq. (17) behaves as $\left(U n_{0} / 2 \epsilon_{\mathbf{q}}\right)^{1 / 2}$. One can also prove that $\epsilon_{\mathbf{q}}$ $\leqslant 4 \pi^{2}|\mathbf{q}|^{2} t$. This means that

$$
\int_{-1 / 2}^{1 / 2} d \mathbf{q} \frac{\epsilon_{\mathbf{q}}+U n_{0}}{\sqrt{\epsilon_{\mathbf{q}}^{2}+2 U \epsilon_{\mathbf{q}} n_{0}}} \geqslant \frac{1}{2 \pi} \sqrt{\frac{U n_{0}}{2 t}} \int_{-1 / 2}^{1 / 2} \frac{d \mathbf{q}}{\mathbf{q} \mid} .
$$

The integral at the right-hand side of Eq. (18) can be done analytically in two dimensions and numerically in three dimensions. When we call the result of the integration in $d$ dimensions $I_{d}$, we see that Eq. (17), for $U / t \rightarrow \infty$, reduces to

$$
n \approx n_{0}+\frac{1}{4 \pi} \sqrt{\frac{U n_{0}}{2 t}} I_{d}-\frac{1}{2},
$$

where $I_{2}=2.22322$ and $I_{3}=2.38008$. This is a quadratic equation in $\sqrt{n_{0}}$, which always yields a positive solution for $n_{0}$ given by

$$
n_{0}=\left(\frac{1}{2} \sqrt{\frac{I_{d}^{2}}{16 \pi^{2}} \frac{U}{2 t}+4 n+2}-\frac{I_{d}}{8 \pi} \sqrt{\frac{U}{2 t}}\right)^{2} .
$$

We can correct for the error we made in Eq. (18) by using a higher value for $I_{d}$, but while this may change the value of $n_{0}$, it will still yield a positive solution. We see from Eq. (20) that $\lim _{U / t \rightarrow \infty} n_{0}=0$ as expected, so we can conclude that the Bogoliubov approximation, as described above, does not predict the phase transition to the Mott insulator phase in two and three dimensions. The reason for this is that the Bogoliubov approach only approximately treats the interactions. As a result, the Bogoliubov approach cannot describe large depletions of the condensate.

We also see from Eq. (18) that in one dimension $I_{1}$ diverges. Substituting this in Eq. (19), we see that there are no Bose-condensed solutions, i.e., solutions with $n_{0} \neq 0$, in one dimension. This is in accordance with the Mermin-WagnerHohenberg theorem [10-12]. 
As the Bogoliubov approximation fails to predict the phase transition to the Mott insulator phase, we now consider a different mean-field theory that treats the interactions exactly and approximates the kinetic energy of the atoms in the optical lattice.

\section{DECOUPLING APPROXIMATION}

To arrive at a mean-field approach, that is capable of describing the Mott insulating phase, we start again from Eq. (1). Analogous to the Bogoliubov approach, we introduce the superfluid order parameter $\psi=\sqrt{n_{i}}=\left\langle c_{i}^{\dagger}\right\rangle=\left\langle c_{i}\right\rangle$, where $n_{i}$ is the expectation value of the number of particles on site $i$. Note that we take the expectation values to be real, as before. We now, however, construct a consistent mean-field theory by substituting

$$
c_{i}^{\dagger} c_{j}=\left\langle c_{i}^{\dagger}\right\rangle c_{j}+c_{i}^{\dagger}\left\langle c_{j}\right\rangle-\left\langle c_{i}^{\dagger}\right\rangle\left\langle c_{j}\right\rangle=\psi\left(c_{i}^{\dagger}+c_{j}\right)-\psi^{2},
$$

into Eq. (1). Performing the substitution yields

$$
\begin{aligned}
H^{\mathrm{eff}}= & -z t \psi \sum_{i}\left(c_{i}^{\dagger}+c_{i}\right)+z t \psi^{2} N_{s}+\frac{1}{2} U \sum_{i} c_{i}^{\dagger} c_{i}^{\dagger} c_{i} c_{i} \\
& -\mu \sum_{i} c_{i}^{\dagger} c_{i},
\end{aligned}
$$

where $z=2 d$ is again the number of nearest-neighbor sites and $N_{s}$ is the total number of lattice sites, as before. This Hamiltonian is diagonal with respect to the site index $i$, so we can use an effective onsite Hamiltonian. If we introduce $\bar{U}=U / z t, \bar{\mu}=\mu / z t$, and the number operator $\hat{n}_{i}=c_{i}^{\dagger} c_{i}$, we find

$$
H_{i}^{\mathrm{eff}}=\frac{1}{2} \bar{U} \hat{n}_{i}\left(\hat{n}_{i}-1\right)-\bar{\mu} \hat{n}_{i}-\psi\left(c_{i}^{\dagger}+c_{i}\right)+\psi^{2},
$$

which is valid on each site $i$. We will therefore drop the subscript $i$ in the following. Note that we scaled all the energies by a factor $1 / z t$, making this Hamiltonian a dimensionless operator.

After writing Eq. (23) in matrix form with respect to an occupation number basis, we can solve the problem numerically by explicitly diagonalizing the part of the matrix with occupation number below a certain maximum value [9]. Later we also follow this procedure, but we first determine the phase diagram analytically using second-order perturbation theory.

\section{A. Second-order perturbation theory}

When we write $H^{\mathrm{eff}}=H^{(0)}+\psi V$, with

$$
\begin{gathered}
H^{(0)}=\frac{1}{2} \bar{U} \hat{n}(\hat{n}-1)-\bar{\mu} \hat{n}+\psi^{2}, \\
V=-\left(c^{\dagger}+c\right),
\end{gathered}
$$

we see that in an occupation number basis the odd powers of the expansion of the energy in $\psi$ will always be zero. If we denote the unperturbed energy of the state with exactly $n$ particles by $E_{n}^{(0)}$, we find that the unperturbed ground-state energy is given by

$$
E_{g}^{(0)}=\left\{E_{n}^{(0)} \mid n=0,1,2, \ldots\right\}_{\min } .
$$

Comparing $E_{n}^{(0)}$ and $E_{n+1}^{(0)}$ yields

$$
E_{g}^{(0)}= \begin{cases}0 & \text { if } \bar{\mu}<0, \\ \frac{1}{2} \bar{U} g(g-1)-\bar{\mu} g & \text { if } \bar{U}(g-1)<\bar{\mu}<\bar{U} g .\end{cases}
$$

Next, we calculate the second-order correction to the energy with the well-known expression

$$
E_{g}^{(2)}=\psi^{2} \sum_{n \neq g} \frac{|\langle g|V| n\rangle|^{2}}{E_{g}^{(0)}-E_{n}^{(0)}},
$$

where $|n\rangle$ denotes the unperturbed wave function with $n$ particles, of which the state with $n=g$ particles is the ground state. Since the interaction $V$ couples only to states with one more or one less atom than in the ground state, we find

$$
E_{g}^{(2)}=\frac{g}{\bar{U}(g-1)-\bar{\mu}}+\frac{g+1}{\bar{\mu}-\bar{U} g} .
$$

If we now follow the usual Landau procedure for secondorder phase transitions by writing the ground-state energy as an expansion in $\psi$

$$
E_{g}(\psi)=a_{0}(g, \bar{U}, \bar{\mu})+a_{2}(g, \bar{U}, \bar{\mu}) \psi^{2}+\mathcal{O}\left(\psi^{4}\right),
$$

and minimize it as a function of the superfluid order parameter $\psi$, we find that $\psi=0$ when $a_{2}(g, \bar{U}, \bar{\mu})>0$ and that $\psi$ $\neq 0$ when $a_{2}(g, \bar{U}, \bar{\mu})<0$. This means that $a_{2}(g, \bar{U}, \bar{\mu})=0$ signifies the boundary between the superfluid and the insulator phases. Solving

$$
a_{2}(g, \bar{U}, \bar{\mu})=\frac{g}{\bar{U}(g-1)-\bar{\mu}}+\frac{g+1}{\bar{\mu}-\bar{U} g}+1=0,
$$

yields

$$
\bar{\mu}_{ \pm}=\frac{1}{2}[\bar{U}(2 g-1)-1] \pm \frac{1}{2} \sqrt{\bar{U}^{2}-2 \bar{U}(2 g+1)+1},
$$

where the subscript \pm denotes the upper and lower halves of the Mott insulating regions of phase space. Note that this result is exact within our mean-field theory. Figure 2 shows a plot of Eq. (29) for $g=1,2,3$. By equating $\bar{\mu}_{+}$and $\bar{\mu}_{-}$we can find the point of smallest $\bar{U}$ for each lobe. Denoting this critical value of $\bar{U}$ by $\bar{U}_{c}$ we have

$$
\bar{U}_{c}=2 g+1+\sqrt{(2 g+1)^{2}-1},
$$




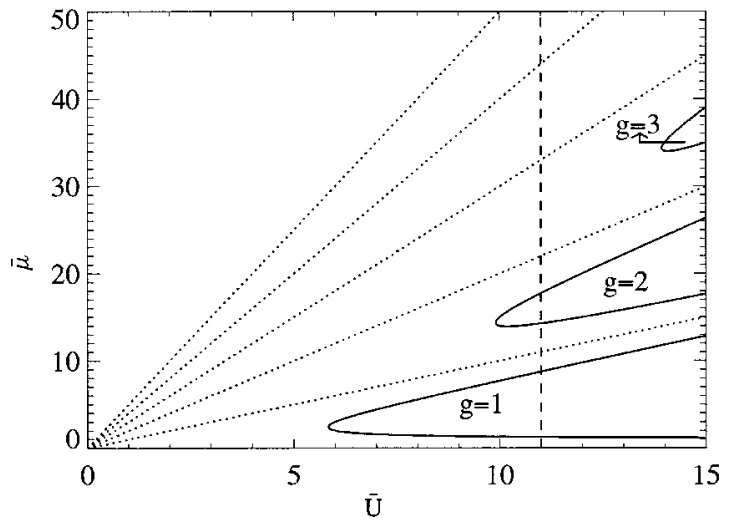

FIG. 2. Phase diagram of the Bose-Hubbard Hamiltonian as obtained from second-order perturbation theory (solid lines). The vertical axis shows the dimensionless chemical potential $\bar{\mu}=\mu / z$ t and the horizontal axis shows the dimensionless interaction strength $\bar{U}=U / z t$. The dotted lines indicate the zeroth-order phase diagram. Later on, Fig. 5 is taken along the dashed line in this figure.

which yields $\bar{U}_{c} \approx 5.83$ for the $g=1$ insulator, a value also found by Ref. [9].

\section{B. Fourth-order perturbation theory}

To find out more about the phase transition, we now carry out fourth-order perturbation theory to find the rate with which the particle density increases as a function of $\bar{\mu}$. In Appendix A, we present a way to calculate the higher-order terms in the perturbation series. Using this procedure we can write the ground-state energy as

$$
E_{g}(\psi)=a_{0}(g, \bar{U}, \bar{\mu})+a_{2}(g, \bar{U}, \bar{\mu}) \psi^{2}+a_{4}(g, \bar{U}, \bar{\mu}) \psi^{4},
$$

with

$$
\begin{aligned}
a_{4}(g, \bar{U}, \bar{\mu})= & \frac{g(g-1)}{[\bar{U}(g-1)-\bar{\mu}]^{2}[\bar{U}(2 g-3)-2 \bar{\mu}]} \\
& +\frac{(g+1)(g+2)}{(\bar{\mu}-\bar{U} g)^{2}[2 \bar{\mu}-\bar{U}(2 g+1)]} \\
& -\left(\frac{g}{\bar{U}(g-1)-\bar{\mu}}+\frac{g+1}{\bar{\mu}-\bar{U} g}\right) \\
& \times\left(\frac{g}{[\bar{U}(g-1)-\bar{\mu}]^{2}}+\frac{g+1}{(\bar{\mu}-\bar{U} g)^{2}}\right) .
\end{aligned}
$$

In Figs. 3(a) and 3(b) we show plots of Eq. (31) together with the result of an exact numerical diagonalization of the effective Hamiltonian. As can be seen, the overlap is very good near the boundary given by Eq. (29). In Fig. 4 it can be seen that the numerical result exhibits a cusp when $\bar{U}=\bar{\mu}$, which is not predicted by Eq. (31). This is due to the fact that in this particular case we need to use first-order degenerate
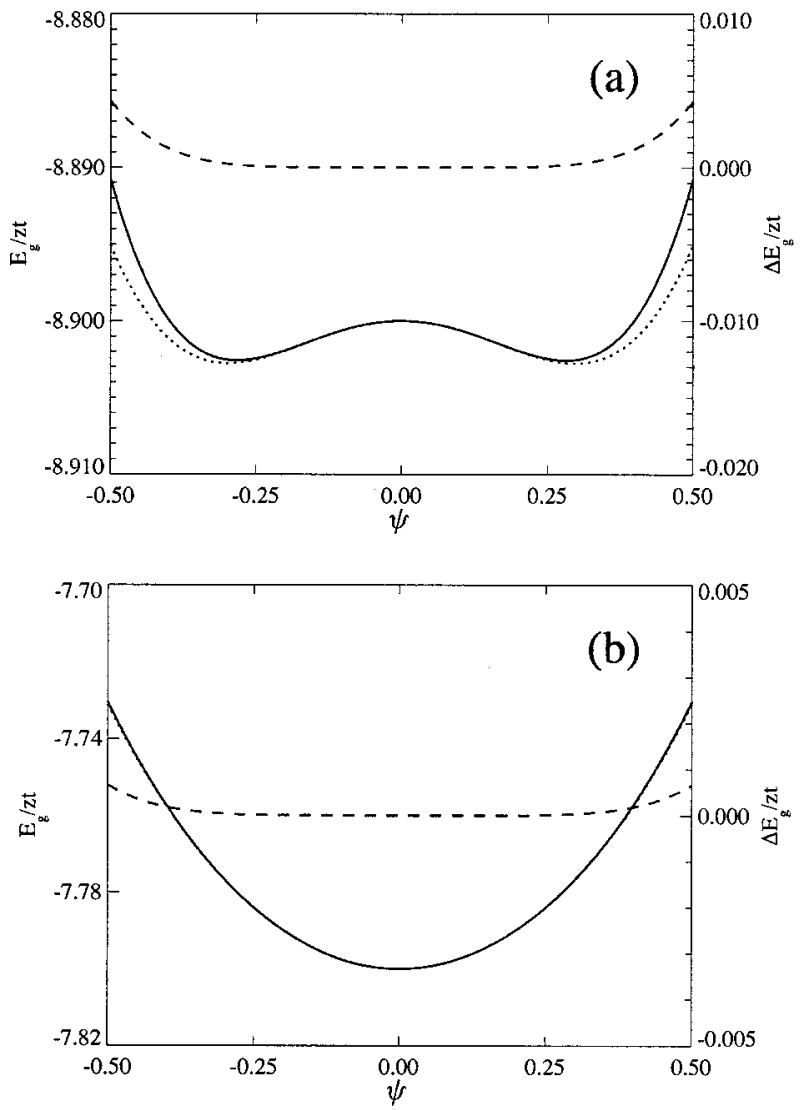

FIG. 3. Ground-state energy (in units of the hopping energy $z t$ ) as a function of $\psi$ for (a) $\bar{U}=11$ and $\bar{\mu}=8.9$ and for (b) $\bar{U}=11$ and $\bar{\mu}=7.8$. The solid line represents fourth-order perturbation theory whereas the dotted line represents a numerical diagonalization of the effective hamiltonian. The dashed line is the difference between the two (scale on the right).

perturbation theory, because at $\bar{\mu}=n \bar{U}$ the states with $n-1$ and $n$ particles per site form a doubly degenerate ground state. The resulting expression for the ground-state energy is now nonanalytic and given by

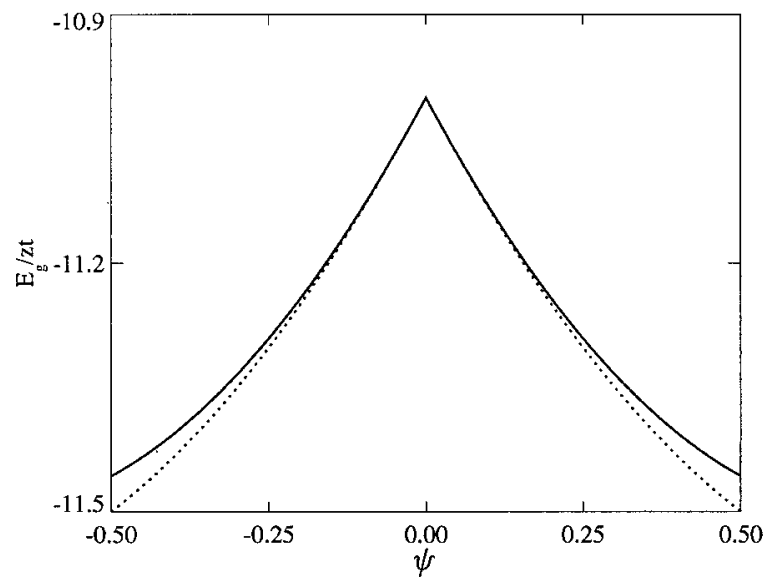

FIG. 4. Ground-state energy (in units of the hopping energy $z t$ ) as a function of $\psi$ for $\bar{U}=\bar{\mu}=11$, as obtained from first-order perturbation theory (solid line) and from numerical diagonalization of the effective hamiltonian (dotted line). 


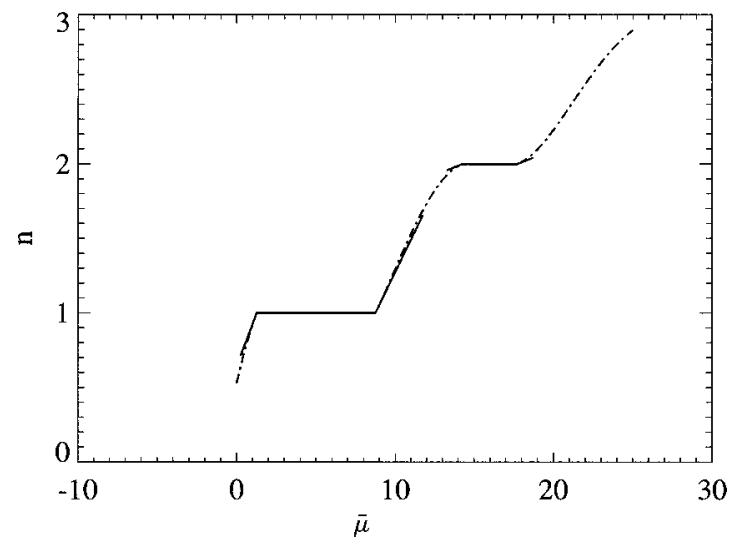

FIG. 5. The density as a function of dimensionless chemical potential $\bar{\mu}=\mu / z t$ for a dimensionless interaction strength of $\bar{U}$ $=U / z t=11$, i.e., along the dashed line in Fig. 2.

$$
\left.E_{g}(\psi)\right|_{\bar{\mu}=n \bar{U}}=-\frac{1}{2} \bar{U} n(n+1)+\psi^{2}-|\psi| \sqrt{n+1},
$$

which is the solid line in Fig. 4. Note that the occurrence of a cusp is analogous to the well-known Jahn-Teller effect in solid-state physics [13].

We now continue by calculating the average number of particles per site in the grand-canonical ensemble by

$$
n=-\frac{\partial\left\langle H^{\mathrm{eff}}\right\rangle}{\partial \mu}=-\frac{\partial E_{g}\left(\psi=\psi_{\min }\right)}{\partial \bar{\mu}}=g-\frac{\partial}{\partial \bar{\mu}}\left(\frac{a_{2}(g, \bar{U}, \bar{\mu})^{2}}{4 a_{4}(g, \bar{U}, \bar{\mu})}\right),
$$

where $\psi_{\min }=\left[-a_{2}(g, \bar{U}, \bar{\mu}) / 2 a_{4}(g, \bar{U}, \bar{\mu})\right]^{1 / 2}$ is the minimum of Eq. (31). Making use of the previous results, we can now plot the density as a function of $\bar{\mu}$ for a fixed value of $\bar{U}$. Between the edges $\bar{\mu}_{ \pm}$, the density will remain constant because $\psi_{\min }=0$ and the second term in the right-hand side of Eq. (34) does not contribute. Outside that region, the density will start to change with a nonzero slope. In Fig. 5 this is plotted for $\bar{U}=11$. The solid line shows the result of the calculation described above and the dash-dotted line is a numerical result obtained by exact diagonalization. As can be seen, the analytical results are in good agreement with the numerical calculation. We can now also qualitatively understand the solution found numerically by Jaksch et al. [5] for an optical lattice in an external harmonic trap. In a first approximation, we can describe the effect of a slowly varying trapping potential by substituting $\bar{\mu}$ in Eq. (34) by $\bar{\mu}^{\prime}=\bar{\mu}$ $+V(\mathbf{r})$, where $V(\mathbf{r})$ is the external trapping potential. Combining this with Fig. 5 yields the density profile found in Ref. $[5]$.

\section{DISPERSION RELATIONS}

An important property of the Mott insulating phase is that the fluctuation in the average number of particles per site goes to zero at zero temperature. Since these fluctuations can be described as quasiparticle and quasihole excitations, we will study these now. We calculate the quasiparticle and quasihole dispersions using a functional integral formalism. We start by deriving an expression for the effective action. Readers unfamiliar with functional integrals may want to skip to Sec. IV B, where we discuss the results of the calculation.

\section{A. The effective action}

We define complex functions $a_{i}^{*}(\tau)$ and $a_{i}(\tau)$, respectively, and write the grand-canonical partition function as

$$
Z=\operatorname{Tr} e^{-\beta \hat{H}}=\int \mathcal{D} a^{*} \mathcal{D} a \exp \left\{-S\left[a^{*}, a\right] / \hbar\right\},
$$

where the action $S\left[a^{*}, a\right]$ is given by

$$
\begin{aligned}
S\left[a^{*}, a\right]= & \int_{0}^{\hbar \beta} d \tau\left[\sum_{i} a_{i}^{*}\left(\hbar \frac{\partial}{\partial \tau}-\mu\right) a_{i}-\sum_{i j} t_{i j} a_{i}^{*} a_{j}\right. \\
& \left.+\frac{1}{2} U \sum_{i} a_{i}^{*} a_{i}^{*} a_{i} a_{i}\right]
\end{aligned}
$$

with $\beta=1 / k_{B} T, k_{B}$ the Boltzmann constant, and $T$ the temperature. To decouple the hopping term, we perform a Hubbard-Stratonovich transformation by adding a complete square to the action, which then becomes

$$
\begin{aligned}
S\left[a^{*}, a, \psi^{*}, \psi\right]= & S\left[a^{*}, a\right]+\int_{0}^{\hbar \beta} d \tau \sum_{i j}\left(\psi_{i}^{*}-a_{i}^{*}\right) t_{i j} \\
& \times\left(\psi_{j}-a_{j}\right)
\end{aligned}
$$

Here $\psi^{*}$ and $\psi$ are the order parameter fields. To obtain an effective action as a function of these fields, we rewrite Eq. (37) as

$$
\begin{aligned}
S\left[a^{*}, a, \psi^{*}, \psi\right]= & \int_{0}^{\hbar \beta} d \tau\left[\sum_{i} a_{i}^{*}\left(\hbar \frac{\partial}{\partial \tau}-\mu\right) a_{i}\right. \\
& +\frac{1}{2} U \sum_{i} a_{i}^{*} a_{i}^{*} a_{i} a_{i}-\sum_{i j} t_{i j}\left(a_{i}^{*} \psi_{j}\right. \\
& \left.\left.+\psi_{i}^{*} a_{j}\right)+\sum_{i j} t_{i j} \psi_{i}^{*} \psi_{j}\right]
\end{aligned}
$$

and integrate over the original fields $a_{i}^{*}$ and $a_{i}$. If we denote by $S^{(0)}\left[a^{*}, a\right]$ the action for $t_{i j}=0$, we have explicitly that

$$
\begin{aligned}
\exp (- & \left.S^{\operatorname{eff}}\left[\psi^{*}, \psi\right] / \hbar\right) \\
\equiv & \exp \left(-\frac{1}{\hbar} \int_{0}^{\hbar \beta} d \tau \sum_{i j} t_{i j} \psi_{i}^{*} \psi_{j}\right) \int \mathcal{D} a^{*} \mathcal{D} a \\
& \times \exp \left\{-S^{(0)}\left[a^{*}, a\right] / \hbar\right\} \\
& \times \exp \left[-\frac{1}{\hbar} \int_{0}^{\hbar \beta} d \tau\left(-\sum_{i j} t_{i j}\left(a_{i}^{*} \psi_{j}+\psi_{i}^{*} a_{j}\right)\right)\right] .
\end{aligned}
$$

We can now calculate $S^{\text {eff }}$ perturbatively by Taylor expanding the exponent in the integrand of Eq. (39) and evalu- 
ating the various correlation functions of the field theory given by $S^{(0)}$. This yields for the quadratic part of the effective action

$$
\begin{aligned}
S^{(2)}\left[\psi^{*}, \psi\right] & =-\frac{1}{2 \hbar}\left\langle\left(\int_{0}^{\hbar \beta} d \tau \sum_{i j} t_{i j}\left(a_{i}^{*} \psi_{j}+\psi_{i}^{*} a_{j}\right)\right)^{2}\right\rangle_{S^{(0)}}+\int_{0}^{\hbar \beta} d \tau \sum_{i j} t_{i j} \psi_{i}^{*} \psi_{j} \\
& =-\frac{1}{2 \hbar}\left\langle\int_{0}^{\hbar \beta} \int_{0}^{\hbar \beta} d \tau d \tau^{\prime} \sum_{i j i^{\prime} j^{\prime}} t_{i j} t_{i^{\prime} j^{\prime}}\left(a_{i}^{*} \psi_{j}+\psi_{i}^{*} a_{j}\right)\left(a_{i^{\prime}}^{*} \psi_{j^{\prime}}+\psi_{i^{\prime}}^{*} a_{j^{\prime}}\right)\right\rangle_{S^{(0)}}+\int_{0}^{\hbar \beta} d \tau \sum_{i j} t_{i j} \psi_{i}^{*} \psi_{j} .
\end{aligned}
$$

If we perform the multiplication in the first term on the righthand side and use the information we have about the correlations in the unperturbed system, i.e.,

$$
\begin{gathered}
\left\langle a_{i}^{*} a_{j}^{*}\right\rangle_{S^{(0)}}=\left\langle a_{i} a_{j}\right\rangle_{S^{(0)}}=0, \\
\left\langle a_{i}^{*} a_{j}\right\rangle_{S^{(0)}}=\left\langle a_{i} a_{j}^{*}\right\rangle_{S^{(0)}}=\left\langle a_{i} a_{i}^{*}\right\rangle_{S^{(0)}} \delta_{i, j},
\end{gathered}
$$

we obtain in the first instance

$$
\begin{aligned}
S^{(2)}\left[\psi^{*}, \psi\right]= & \int_{0}^{\hbar \beta} d \tau\left\{\sum_{i j} t_{i j} \psi_{i}^{*}(\tau) \psi_{j}(\tau)\right. \\
& -\frac{1}{\hbar} \int_{0}^{\hbar \beta} d \tau^{\prime} \sum_{i j i^{\prime} j^{\prime}} t_{i j} t_{i^{\prime} j^{\prime}} \psi_{j}^{*}(\tau) \\
& \left.\times\left\langle a_{i}(\tau) a_{i^{\prime}}^{*}\left(\tau^{\prime}\right)\right\rangle_{S^{(0)}} \psi_{j^{\prime}}\left(\tau^{\prime}\right)\right\},
\end{aligned}
$$

where we have now shown the $\tau$ dependence of the fields explicitly for clarity reasons. Because we will only consider nearest-neighbor hopping, we write

$$
t_{i j}=t_{j i}=\left\{\begin{array}{cc}
t & \text { for nearest neighbors } \\
0 & \text { otherwise. }
\end{array}\right.
$$

First we treat the part of Eq. (42) that is linear in $t_{i j}$. We have

$$
\sum_{i j} t_{i j} \psi_{i}^{*}(\tau) \psi_{j}(\tau)=\sum_{i} t \psi_{i}^{*}(\tau) \psi_{i \pm\{1\}}(\tau),
$$

where $\pm\{1\}$ denotes all possible jumps to nearest neighbors. In the case of one dimension this would simply be \pm 1 . If we call the lattice spacing $a$ and introduce cartesian momentum components $k_{i}$ with $i=1, \ldots, d$, where $d$ is again the number of dimensions, we find

$$
\sum_{i j} t_{i j} \psi_{i}^{*}(\tau) \psi_{j}(\tau)=\sum_{\mathbf{k}} 2 t \psi_{\mathbf{k}}(\tau) \psi_{\mathbf{k}}^{*}(\tau) \sum_{j=1}^{d} \cos \left(k_{j} a\right) .
$$

Next we calculate the part that is quadratic in $t_{i j}$. We can treat this part by looking at double jumps. The expectation value of $\left\langle a_{i} a_{i^{\prime}}^{*}\right\rangle_{S^{(0)}}$ is proportional $\delta_{i i}$, and independent of the site $i$, according to Eq. (41). This means that we find, with similar notation as before,

$$
\begin{aligned}
\sum_{j i^{\prime} j^{\prime}} t_{i j} t_{i^{\prime} j^{\prime}} \psi_{j}^{*}(\tau)\left\langle a_{i}(\tau) a_{i^{\prime}}^{*}\left(\tau^{\prime}\right)\right\rangle_{S^{(0)}} \psi_{j^{\prime}}\left(\tau^{\prime}\right) \\
=\left\langle a_{i}(\tau) a_{i}^{*}\left(\tau^{\prime}\right)\right\rangle_{S^{(0)}} \sum_{j j^{\prime}} t_{i j^{\prime}} t_{i j^{\prime}} \psi_{j}^{*}(\tau) \psi_{j^{\prime}}\left(\tau^{\prime}\right) \\
=t^{2}\left\langle a_{i}(\tau) a_{i}^{*}\left(\tau^{\prime}\right)\right\rangle_{S^{(0)}} \sum_{j}\left\{z \psi_{j}^{*}(\tau) \psi_{j}\left(\tau^{\prime}\right)\right. \\
\left.\quad+\psi_{j}^{*}(\tau) \psi_{j \pm\{2\}}\left(\tau^{\prime}\right)+\psi_{j}^{*}(\tau) \psi_{j \pm\{\sqrt{2}\}}\left(\tau^{\prime}\right)\right\},
\end{aligned}
$$

with $z$ again the number of nearest neighbors. The first term in the summand is a jump in each direction, followed by a jump back. The second term indicates two jumps in the same direction and the third term is a jump in each direction followed by a jump in a perpendicular direction. Note that the third term is absent in one dimension. It can be shown that the complete double jump term reduces to

$$
\begin{aligned}
& \sum_{j i^{\prime} j^{\prime}} t_{i j} t_{i^{\prime} j^{\prime}} \psi_{j}^{*}(\tau)\left\langle a_{i}(\tau) a_{i^{\prime}}^{*}\left(\tau^{\prime}\right)\right\rangle_{S^{(0)}} \psi_{j^{\prime}}\left(\tau^{\prime}\right) \\
& =\left\langle a_{i}(\tau) a_{i}^{*}\left(\tau^{\prime}\right)\right\rangle_{S^{(0)}} \sum_{\mathbf{k}} \psi_{\mathbf{k}}^{*}(\tau) \psi_{\mathbf{k}}\left(\tau^{\prime}\right) \bar{\epsilon}_{\mathbf{k}}^{2}
\end{aligned}
$$

where we again used $\bar{\epsilon}_{\mathbf{k}}=2 t \sum_{j=1}^{d} \cos \left(k_{j} a\right)$.

To also treat the time dependence of the fields, we introduce Matsubara frequencies $\hbar \omega_{n}=\pi(2 n) / \hbar \beta$ by

$$
\begin{aligned}
& \psi_{\mathbf{k}}(\tau)=\sum_{n} \frac{1}{\sqrt{\hbar \beta}} \psi_{\mathbf{k} n} e^{-i \omega_{n} \tau} \\
& \psi_{\mathbf{k}}^{*}(\tau)=\sum_{n} \frac{1}{\sqrt{\hbar \beta}} \psi_{\mathbf{k} n}^{*} e^{+i \omega_{n} \tau} .
\end{aligned}
$$

To translate the expectation value of the fields into the expectation value of operators, we introduce an (imaginary) time-ordering operator $\mathrm{T}$. As a result

$$
\left\langle a_{i}(\tau) a_{i^{\prime}}^{*}\left(\tau^{\prime}\right)\right\rangle_{S^{(0)}}=\left\langle\mathrm{T}\left[a_{i}(\tau) a_{i^{\prime}}^{\dagger}\left(\tau^{\prime}\right)\right]\right\rangle_{S^{(0)}} .
$$

The time ordering can easily be expressed in Heavyside functions as 


$$
\begin{aligned}
\left\langle\mathrm{T}\left[a_{i}(\tau) a_{i^{\prime}}^{\dagger}\left(\tau^{\prime}\right)\right]\right\rangle_{S^{(0)}}= & \theta\left(\tau-\tau^{\prime}\right)\left\langle a_{i}(\tau) a_{i^{\prime}}^{\dagger}\left(\tau^{\prime}\right)\right\rangle_{S^{(0)}} \\
& +\theta\left(\tau^{\prime}-\tau\right)\left\langle a_{i^{\prime}}^{\dagger}\left(\tau^{\prime}\right) a_{i}(\tau)\right\rangle_{S^{(0)}}
\end{aligned}
$$

If we use the unperturbed energies as given by Eq. (25), we thus find

$$
\begin{aligned}
\left\langle a_{i}(\tau) a_{i^{\prime}}^{*}\left(\tau^{\prime}\right)\right\rangle_{S^{(0)}}= & \theta\left(\tau-\tau^{\prime}\right)(1+g) \\
& \times \exp \left\{-\left(E_{g+1}^{(0)}-E_{g}^{(0)}\right)\left(\tau-\tau^{\prime}\right) / \hbar\right\} \\
& +\theta\left(\tau^{\prime}-\tau\right) g \exp \left\{\left(E_{g-1}^{(0)}-E_{g}^{(0)}\right)\right. \\
& \left.\times\left(\tau-\tau^{\prime}\right) / \hbar\right\} .
\end{aligned}
$$

Because $g$ minimizes $E_{g}^{(0)}$ we know that

$$
\begin{gathered}
E_{g+1}^{(0)}-E_{g}^{(0)}=-\mu+g U>0, \\
E_{g}^{(0)}-E_{g-1}^{(0)}=-\mu+(g-1) U<0 .
\end{gathered}
$$

Note that we use parameters $\mu$ and $U$ instead of $\bar{\mu}$ and $\bar{U}$, because we have not yet divided out the factor $z t$. Combining the above with Eq. (42) we find

$$
\begin{aligned}
S^{(2)}\left[\psi^{*}, \psi\right]= & \sum_{n} \sum_{\mathbf{k}}\left|\psi_{\mathbf{k} n}\right|^{2} \bar{\epsilon}_{\mathbf{k}} \\
& \times\left(1-\frac{\bar{\epsilon}_{\mathbf{k}}}{\hbar} \int_{-\infty}^{0} d \tau^{\prime}(1+g)\right. \\
& \times \exp \left\{\left(-i \hbar \omega_{n}-\mu+g U\right) \tau^{\prime} / \hbar\right\} \\
& -\frac{\bar{\epsilon}_{\mathbf{k}}}{\hbar} \int_{0}^{\infty} d \tau^{\prime} g \exp \left\{-\left(i \hbar \omega_{n}+\mu\right.\right. \\
& \left.\left.-(g-1) U) \tau^{\prime} / \hbar\right\}\right) .
\end{aligned}
$$

Performing the $\tau^{\prime}$ integration we then easily obtain

$$
\begin{aligned}
S^{(2)}\left[\psi^{*}, \psi\right]= & \sum_{n} \sum_{\mathbf{k}}\left|\psi_{\mathbf{k} n}\right|^{2} \bar{\epsilon}_{\mathbf{k}} \times\left[1-\bar{\epsilon}_{\mathbf{k}}\left(\frac{g+1}{-i \hbar \omega_{n}-\mu+g U}\right.\right. \\
& \left.\left.+\frac{g}{i \hbar \omega_{n}+\mu-(g-1) U}\right)\right]
\end{aligned}
$$

Note that this result is exact within our mean-field theory. It contains all powers of the frequencies and momenta and no gradient expansion has been applied. This is important because the elementary excitations are gapped as we will show in the next section.

We can find an equation for real energies $\hbar \omega$ by substituting $i \omega_{n} \rightarrow \omega$ and equating the remaining factor to zero. This gives

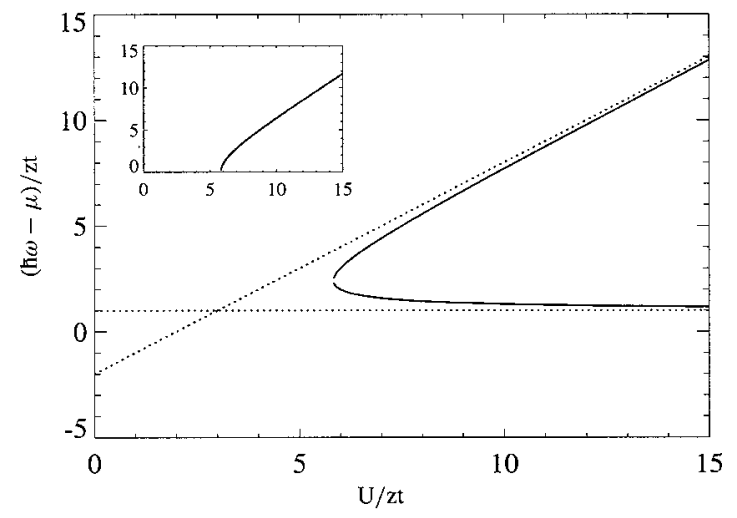

FIG. 6. The quasiparticle and the quasihole energy (in units of the hopping energy $z t$ ) for $\mathbf{k}=0$ in the $g=1$ insulator lobe. The dotted lines are the asymptotes of the curves. The inset shows the resulting first-order approximation to the dispersion of the density fluctuations.

$$
0=\left[1-\bar{\epsilon}_{\mathbf{k}}\left(\frac{g+1}{-\hbar \omega-\mu+g U}+\frac{g}{\hbar \omega+\mu-(g-1) U}\right)\right] .
$$

Ultimately this yields the result Eq. (56) given below in Sec. IV B.

\section{B. Results}

Now we will explore the results of the calculation presented in the previous section. The quasiparticle and quasihole dispersions are given by

$$
\begin{aligned}
\hbar \omega_{q p, q h}= & -\mu+\frac{U}{2}(2 g-1)-\frac{\bar{\epsilon}_{\mathbf{k}}}{2} \\
& \pm \frac{1}{2} \sqrt{\bar{\epsilon}_{\mathbf{k}}^{2}-(4 g+2) U \bar{\epsilon}_{\mathbf{k}}+U^{2}} .
\end{aligned}
$$

In Fig. 6(a) we show for $\mathbf{k}=0$ a plot of the above equations. The dotted lines indicate the asymptotes of Eq. (56), which are given by

$$
\begin{aligned}
\lim _{U \rightarrow \infty} \hbar \omega_{q p} & =-\mu+g U-(g+1) \bar{\epsilon}_{\mathbf{0}} \\
& =E_{g+1}^{(0)}-E_{g}^{(0)}-(g+1) z t, \\
\lim _{U \rightarrow \infty} \hbar \omega_{q h} & =-\mu+(g-1) U+g \overline{\boldsymbol{\epsilon}}_{\mathbf{0}} \\
& =E_{g}^{(0)}-E_{g-1}^{(0)}+g z t,
\end{aligned}
$$

with $E_{g+1}^{(0)}-E_{g}^{(0)}$ and $E_{g}^{(0)}-E_{g-1}^{(0)}$ given by Eq. (52). The difference between Eq. (57) and Eq. (52) is caused by the fact that Eq. (52) is calculated for $t=0$. It can easily be understood that for $t \neq 0$, the first-order correction is due to the hopping terms $c_{j}^{\dagger} c_{i} t$, where site $j$ is one of the nearest neighbors of site $i$. When we have $g$ particles in all lattice sites and we add one particle to site $i$, we have $\left\langle c_{j}^{\dagger} c_{i}\right\rangle=g$ +1 , so the effective hopping parameter for a particle is $(g$ 


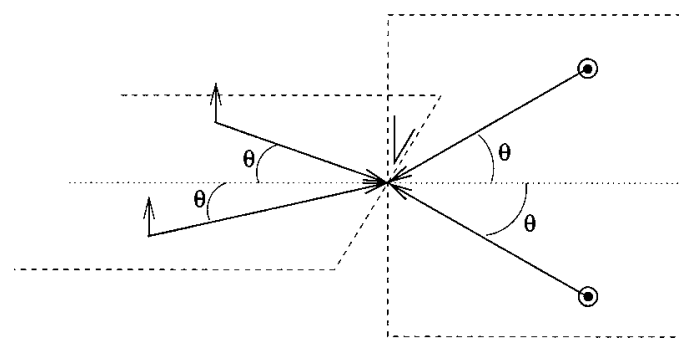

FIG. 7. Laser beam configuration for a three-dimensional optical lattice.

$+1) t$. However, when we remove a particle from site $i$, we have $\left\langle c_{i}^{\dagger} c_{j}\right\rangle=g$, which represents a particle hopping to site $i$ from one of its nearest neighbors. The effective hopping parameter for a hole is therefore only $g t$. In combination, we see that in the limit of $U \rightarrow \infty$, Eq. (56) indeed reduces to a physically intuitive result.

As shown above, the slopes of the asymptotes differ exactly by $U$, so in the limit of $U / z t \rightarrow \infty$ the gap for the creation of a quasiparticle-quasihole pair is equal to $U$. We can find a first approximation for the dispersion of the density fluctuations by subtracting the two solutions, which yields

$$
\epsilon_{\mathbf{k}}=\hbar \omega_{q p}-\hbar \omega_{q h}=\sqrt{\left(\bar{\epsilon}_{\mathbf{k}}\right)^{2}-(4 g+2) U \bar{\epsilon}_{\mathbf{k}}+U^{2}}
$$

In Fig. 6(b) we show again for $\mathbf{k}=0$ a plot of the above equation as a function of $\bar{U}=U / z t$ for $g=1$. We can see that there is a band gap, which proves that the Mott insulator phase is indeed an insulator and we also see that the band gap disappears as we approach the critical value $\bar{U}_{c}$ $=U_{c} / z t \approx 5.83$ that was found earlier. For smaller values of $\bar{U}$ we are in the superfluid phase, which according to the Hugenholtz-Pines theorem is expected to always have gapless density fluctuations.

\section{MICROSCOPIC PARAMETERS}

To estimate the experimental feasibility of observing the described phase transition, we now relate the parameters $t$ and $U$ to the microscopic parameters. Because we have an experimental interest in sodium, we will calculate these parameters for sodium atoms trapped in a lattice constructed with four laser beams. To calculate the hopping parameter $t$, we calculate the overlap between single particle ground-state wave functions in neighboring sites. To calculate the interaction strength $U$, we use the pseudopotential method.

First we calculate the optical potential experienced by the atoms, following the approach of Petsas et al. [14]. We describe a $J=1 / 2 \rightarrow J=3 / 2$ transition and choose a laser beam configuration with two pairs of laser beams. Each pair lies in a plane and the planes are perpendicular to each other. All beams have the same angle $\theta$ with respect to the intersection of the two planes. We choose the quantization axis along the intersection and label it as the $z$ axis. Furthermore, we choose the polarization of the laser beams linear and perpendicular to the plane spanned by the pairs of beams. The configuration is illustrated in Fig. 7. It should be noted that it is also possible to simply superimpose $d$ standing waves to obtain a $d$ dimensional lattice, but this requires stabilization of the relative phases of the laser beams. We define $I_{b}$ as the sum of the intensity of the beams and if $k$ is the magnitude of the $\mathbf{k}$-vector, we define $k_{\perp}=k \sin \theta$ and $k_{\|}=k \cos \theta$. If we add the electric-field components and express them in spherical components

$$
\begin{gathered}
E_{+}=\frac{-1}{\sqrt{2}}\left(E_{x}-i E_{y}\right), \\
E_{-}=\frac{1}{\sqrt{2}}\left(E_{x}+i E_{y}\right), \\
E_{0}=E_{z},
\end{gathered}
$$

we find that the spatial dependence of the intensity of the resulting light field is given by

$$
\begin{gathered}
I_{ \pm} / I_{b}=\frac{1}{2}\left[\cos ^{2}\left(k_{\perp} x\right)+\cos ^{2}\left(k_{\perp} y\right)\right. \\
\left. \pm 2 \cos \left(k_{\perp} x\right) \cos \left(k_{\perp} y\right) \cos \left(2 k_{\|} z\right)\right], \\
I_{0} / I_{b}=0 .
\end{gathered}
$$

Note that at the minima of $I_{ \pm}$the polarization is purely $\sigma^{ \pm}$. Also note that since the linear component is always zero, the two ground-state levels are not coupled.

Following Nienhuis et al. [15] we can now calculate the optical potential. Because of the fact that the ground states are not coupled, we can treat them separately. With $\delta$ the detuning, $\Gamma$ the rate of spontaneous decay and $\Omega_{ \pm}$the Rabi frequencies for the $\sigma^{ \pm}$components of the light field, we can write the potential for the $m_{j}= \pm 1 / 2$ level in the limit of low saturation as

$$
V_{ \pm}=\frac{1}{2} \frac{\hbar \delta}{1+4(\delta / \Gamma)^{2}}\left[\frac{2\left|\Omega_{ \pm}\right|^{2}}{\Gamma^{2}}+\frac{1}{3} \frac{2\left|\Omega_{\mp}\right|^{2}}{\Gamma^{2}}\right],
$$

where the factor $\frac{1}{3}$ arises because of the Clebsch-Gordan coefficients for $J=1 / 2 \rightarrow J=3 / 2$ transitions.

Now we define a convenient prefactor

$$
V_{b}=\frac{1}{2} \frac{\hbar \delta s_{0}}{1+4(\delta / \Gamma)^{2}}=\frac{\hbar \delta s}{2},
$$

where $s=s_{0} /\left[1+4(\delta / \Gamma)^{2}\right]$ is the off-resonance saturation parameter and $s_{0}=2|\Omega|^{2} / \Gamma^{2}$ is the on-resonance saturation parameter, which is usually written as $s_{0}=I / I_{s}$. The satura- 
tion intensity $I_{s}$ is a constant for a given transition. If we substitute Eq. (62) in Eq. (61), we find

$$
\begin{aligned}
V_{ \pm}= & V_{b}\left(\frac{I_{ \pm}}{I_{b}}+\frac{1}{3} \frac{I_{\mp}}{I_{b}}\right) . \\
= & \frac{2}{3} V_{b}\left[\cos ^{2}\left(k_{\perp} x\right)+\cos ^{2}\left(k_{\perp} y\right)\right. \\
& \left. \pm \cos \left(k_{\perp} x\right) \cos \left(k_{\perp} y\right) \cos \left(2 k_{\|} z\right)\right] .
\end{aligned}
$$

We now write the Hamiltonian of a particle in the potential as

$$
H_{\mathrm{opt}}=\frac{p^{2}}{2 m}+V_{ \pm}
$$

and solve the time-independent Schrödinger equation variationally by assuming an isotropic Gaussian wave function and minimizing the energy as a function of width of the Gaussian. If we call $\beta$ the width of the wave function, we can write the normalized wave function as

$$
\Psi(\mathbf{r})=\langle\mathbf{r} \mid \Psi\rangle=\left(\frac{1}{\pi \beta^{2}}\right)^{3 / 4} e^{-|\mathbf{r}|^{2} / 2 \beta^{2}}
$$

We assume we have a spin-polarized sample of atoms, so we can use either the $V_{+}$or the $V_{-}$potential.

For simplicity we now calculate the parameters for a onedimensional lattice. For the lattice configuration in Fig. 7 this gives approximate results, but for a phase stabilized superposition of three standing waves, the results are immediately applicable. In this case, the potential reduces to $V_{ \pm}$ $=2 V_{b}[2 \pm \cos (2 k z)] / 3+\kappa_{\perp}\left(x^{2}+y^{2}\right) / 2$, where the transverse potential is caused by the fact that the laser beam has a finite width. If we assume the wave function is tightly localized in the center of the local potential well, we can approximate the potential as an harmonic potential $V_{+}=2 V_{b}+\kappa \mathbf{r}^{2} / 2$ with $\kappa$ $=-8 V_{b} k^{2} / 3$, where we assume we can adjust the width of the laser such that $\kappa_{\perp} \approx \kappa$. These approximations yield the well-known equations for the width and the level splitting in the potential

$$
\beta=\left(\frac{\hbar^{2}}{m \kappa}\right)^{1 / 4}, \quad \hbar \omega=\sqrt{\kappa / m} .
$$

Using the above width $\beta$ we calculate the value of the interaction strength $U$ with the pseudopotential method. According to Ref. [16] this is valid for sodium even if the width of the trapping volume is of the order of the scattering length. In general the interaction strength between two atoms in the same one-particle wave function is given by

$$
U=\int d \mathbf{r} \int d \mathbf{r}^{\prime} \Psi^{*}(\mathbf{r}) \Psi^{*}\left(\mathbf{r}^{\prime}\right) V_{\mathrm{int}}\left(\mathbf{r}-\mathbf{r}^{\prime}\right) \Psi(\mathbf{r}) \Psi\left(\mathbf{r}^{\prime}\right),
$$

where $V_{\text {int }}\left(\mathbf{r}-\mathbf{r}^{\prime}\right)$ is the interaction potential. If we approximate the potential as

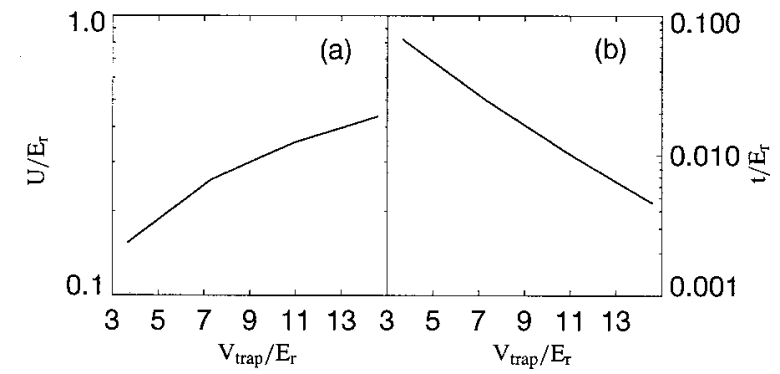

FIG. 8. Plot of (a) $U$ and (b) $t$ as a function of the trap depth. All quantities are in units of the recoil energy $E_{r}$.

$$
V_{\mathrm{int}}\left(\mathbf{r}-\mathbf{r}^{\prime}\right)=\frac{4 \pi a_{s} \hbar^{2}}{m} \delta\left(\mathbf{r}-\mathbf{r}^{\prime}\right)
$$

we can write Eq. (67) as

$$
\begin{aligned}
U & =\frac{4 \pi a_{s} \hbar^{2}}{m} \int d \mathbf{r} \Psi^{*}(\mathbf{r}) \Psi^{*}(\mathbf{r}) \Psi(\mathbf{r}) \Psi(\mathbf{r}) \\
& =\frac{4 \pi a_{s} \hbar^{2}}{m} \int d \mathbf{r}|\Psi(\mathbf{r})|^{4} \\
& =\frac{4 \pi a_{s} \hbar^{2}}{m \beta^{3} \pi^{3 / 2}} \\
& =\frac{2 \hbar \omega}{\sqrt{2 \pi}}\left(\frac{a_{s}}{\beta}\right)
\end{aligned}
$$

where $a_{s}$ is the triplet $s$-wave scattering length. According to Ref. [17] the value of the scattering length for a spinpolarized sodium-sodium collision is $a_{s}=(85 \pm 3) a_{0}$. Note that the use of a one-band model is justified when $U \ll \hbar \omega$, or $\beta \gg 2 a_{s} /(2 \pi)^{1 / 2} \approx 3.5 \mathrm{~nm}$.

Next we calculate the value of the hopping parameter $t$. In the tight-binding limit $t$ is given by

$$
t=-\int d \mathbf{r} \Psi *(\mathbf{r})\left(\frac{p^{2}}{2 m}+V_{ \pm}\right) \Psi\left(\mathbf{r}+a \hat{e}_{j}\right)
$$

where $\hat{e}_{j}$ is an axis vector along a lattice direction, so that when $\Psi(\mathbf{r})$ is the ground-state wave function, $\Psi\left(\mathbf{r}+a \hat{e}_{j}\right)$ is the ground-state wave function of an atom at a neighboring site. One can show that product of two wave functions at neighboring sites is a Gaussian function centered around $\mathbf{r}$ $+a \hat{e}_{j} / 2$. We can therefore approximate the potential around the maximum of the barrier by $V_{ \pm}=2 V_{b} / 3 \mp \kappa \mathbf{r}^{2} / 2$. Substituting this into Eq. (70) ultimately yields

$$
t=\frac{\hbar \omega}{8}\left[1-\left(\frac{2}{\pi}\right)^{2}\right]\left(\frac{a}{\beta}\right)^{2} e^{-\frac{1}{4}(a / \beta)^{2}}
$$

Figures 8(a) and 8(b) show plots of $U / E_{r}$ and $t / E_{r}$ respectively, with $E_{r}=\hbar^{2} k^{2} / 2 m$ the recoil energy. Both are plotted as a function of the trap depth $V_{\text {trap }}=-4 V_{b} / 3$. The values were calculated for a laser wavelength of $600 \mathrm{~nm}$. The satu- 


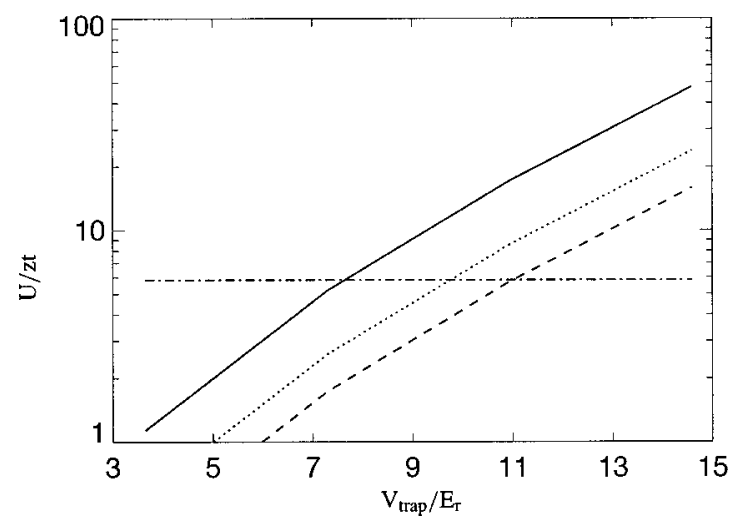

FIG. 9. The dimensionless parameter $U / z t$ plotted as a function of the trap depth (in units of the recoil energy $E_{r}$ ) for one (solid line), two (dotted line), and three dimensions (dashed line). The dash-dotted line is the critical value $\bar{U}=5.83$.

ration parameter needed to reach these trap depths is in the order of $10^{5}$, which is not unrealistic experimentally.

Figure 9 shows also $U / z t$ as a function of the trap depth, for a wavelength of $600 \mathrm{~nm}$, in one, two and three dimensions. Again, the saturation parameter is in the order of $10^{5}$. As has been seen, the desired critical value is reached in all three dimensions. The value of the width $\beta$ lies between $12 \%$ and $8 \%$ of the wavelength in the range considered in the above plots. This implies that both the harmonic approximation and the use of the one-band model are justified.

\section{CONCLUSION}

Due to the absence of the superfluid-insulator phase transition in the Bogoliubov approach, we conclude that the interaction is the dominant component in this phase transition. When the interaction energy is treated exactly, the theory indeed predicts a phase transition. The mean-field theory predicts a phase transition even in one dimension, which we expect to survive as a Kosterlitz-Thouless transition when fluctuations are incorporated. However a definite proof of this requires further study.

We analytically calculated the phase diagram and the particle and hole dispersion relations in the insulator phase. A first-order approximation to the dispersion of the density fluctuations shows that the system indeed goes from a gapped to a gapless phase. A calculation of this dispersion below the critical value for $U / z t$ will have to be done in order to check the presence of linear dispersion that would verify the assumption that the phase with $\psi \neq 0$ is indeed superfluid. The one-band model we used to calculate the parameters for sodium gives optimistic results for future experiments, within the range of parameters it allows.

\section{APPENDIX A: THE PERTURBATION SERIES}

A powerful approach to calculating higher-order terms in the perturbation series is derived in Ref. [18]. Here we only give the result of that derivation. If we denote by $|n\rangle$ the unperturbed wave functions and $E_{n}^{(0)}$ the unperturbed energies, we can define an operator

$$
S_{a}^{k}= \begin{cases}-|a\rangle\langle a| & \text { if } k=0 \\ \sum_{n \neq a} \frac{|n\rangle\langle n|}{\left(E_{a}^{(0)}-E_{n}^{(0)}\right)^{k+1}} & \text { if } k>0,\end{cases}
$$

and one can prove that the $n$th order correction on the energy $E_{a}^{(0)}$ is given by

$$
E_{a}^{(n)}=\operatorname{Tr}\left[\sum_{\{n-1\}} S_{a}^{k_{0}} \hat{V} \ldots \hat{V} S_{a}^{k_{n}}\right],
$$

where $\{n\}=\left\{k_{0}, \cdots, k_{n} \mid k_{0}+\ldots+k_{n}=n\right\}$. In the case of $n$ $=2$, this quickly gives the well-known result

$$
\begin{aligned}
E_{a}^{(2)} & =\operatorname{Tr}\left[\sum_{\{1\}} S_{a}^{k_{0}} \hat{V} S_{a}^{k_{1}} \hat{V} S_{a}^{k_{2}}\right] \\
& =\left\langle a\left|\hat{V} S_{a}^{1} \hat{V}\right| a\right\rangle, \\
& =\sum_{n \neq a} \frac{|\langle n|\hat{V}| a\rangle|^{2}}{\left(E_{a}^{(0)}-E_{n}^{(0)}\right)} .
\end{aligned}
$$

The same can be done for $E_{a}^{(1)}, E_{a}^{(3)}$, and $E_{a}^{(4)}$. The first two can be shown to involve only terms proportional to odd orders of $V$, and with $V \propto\left(c^{\dagger}+c\right)$ these are of course zero. The fourth-order term is in general given by

$$
\begin{aligned}
E_{a}^{(4)}= & \operatorname{Tr}\left[\sum_{\{3\}} S_{a}^{k_{0}} \hat{V} S_{a}^{k_{1}} \hat{V} S_{a}^{k_{2}} \hat{V} S_{a}^{k_{3}} \hat{V} S_{a}^{k_{4}}\right] \\
= & \left\langle a\left|\hat{V} S_{a}^{1} \hat{V} S_{a}^{1} \hat{V} S_{a}^{1} \hat{V}\right| a\right\rangle-\left\langle a\left|\hat{V} S_{a}^{1} \hat{V}\right| a\right\rangle\left\langle a\left|\hat{V} S_{a}^{2} \hat{V}\right| a\right\rangle \\
& -2\langle a|\hat{V}| a\rangle\left\langle a\left|\hat{V} S_{a}^{1} \hat{V} S_{a}^{2} \hat{V}\right| a\right\rangle+\langle a|\hat{V}| a\rangle^{2} \\
& \times\left\langle a\left|\hat{V} S_{a}^{3} \hat{V}\right| a\right\rangle .
\end{aligned}
$$

If we drop the terms containing expectation values of odd powers of $V$ and substitute Eq. (A1), we find

$$
\begin{aligned}
E_{a}^{(4)}= & \left\langle a\left|\hat{V} S_{a}^{1} \hat{V} S_{a}^{1} \hat{V} S_{a}^{1} \hat{V}\right| a\right\rangle-\left\langle a\left|\hat{V} S_{a}^{1} \hat{V}\right| a\right\rangle\left\langle a\left|\hat{V} S_{a}^{2} \hat{V}\right| a\right\rangle \\
= & \sum_{n, p, q \neq a}\langle a|\hat{V}| n\rangle\left(-E_{a}^{(2)} \frac{\langle n|\hat{V}| a\rangle}{\left(E_{a}^{(0)}-E_{n}^{(0)}\right)^{2}}\right. \\
& \left.+\frac{\langle n|\hat{V}| p\rangle}{\left(E_{a}^{(0)}-E_{n}^{(0)}\right)} \frac{\langle p|\hat{V}| q\rangle}{\left(E_{a}^{(0)}-E_{p}^{(0)}\right)} \frac{\langle q|\hat{V}| a\rangle}{\left(E_{a}^{(0)}-E_{q}^{(0)}\right)}\right),
\end{aligned}
$$

which we have used to derive Eq. (32). 
[1] G. Grynberg, B. Lounis, P. Verkerk, J.-Y. Courtois, and C. Salomon, Phys. Rev. Lett. 70, 2249 (1993).

[2] P. S. Jessen and I. H. Deutsch, Adv. At., Mol., Opt. Phys. 37, 95 (1996) and references therein.

[3] A. J. Kerman, V. Vuletić, C. Chin, and S. Chu, Phys. Rev. Lett. 84, 439 (2000).

[4] G. K. Brennen, C. M. Caves, P. S. Jessen, and I. H. Deutsch, Phys. Rev. Lett. 82, 1060 (1999).

[5] D. Jaksch, C. Bruder, J. I. Cirac, C. W. Gardiner, and P. Zoller, Phys. Rev. Lett. 81, 3108 (1998).

[6] N. F. Mott, Proc. Phys. Soc., London, Sect. A 62, 416 (1949).

[7] A. van Otterlo and K.-H. Wagenblast, Phys. Rev. Lett. 72, 3598 (1994).

[8] E. Roddick and D. Stroud, Phys. Rev. B 48, 16600 (1993).

[9] K. Sheshadri, H. R. Krishnamurthy, R. Pandit, and T.V. Ramakrishnan, Europhys. Lett. 22, 257 (1993).

[10] N. D. Mermin, Phys. Rev. 167, 250 (1968).
[11] N. D. Mermin and H. Wagner, Phys. Rev. Lett. 22, 1133 (1966).

[12] P. C. Hohenberg, Phys. Rev. 158, 383 (1967).

[13] H. A. Jahn and E. Teller, Proc. R. Soc. London, Ser. A 164, 117 (1937).

[14] K. I. Petsas, A. B. Coates, and G. Grynberg, Phys. Rev. A 50, 5173 (1994).

[15] G. Nienhuis, P. van der Straten, and S-Q. Shang, Phys. Rev. A 44, 462 (1991).

[16] E. Tiesinga, C. J. Williams, F. H. Mies, and P. S. Julienne, Phys. Rev. A 61, 063416 (2000).

[17] E. Tiesinga, C. J. Williams, P. S. Julienne, K. M. Jones, P. D. Lett, and W. D. Phillips, J. Res. Natl. Inst. Stand. Technol. 101, 505 (1996).

[18] A. Messiah, Quantum Mechanics (North-Holland, Amsterdam, 1961), Vol. 2. 